\title{
Search for a Light Pseudoscalar Particle in the Decay $K_{L}^{0} \rightarrow \pi^{0} \pi^{0} X$ at E391a Experiment
}

\author{
Y. C. Tung ${ }^{* \dagger}$ \\ Department of Physics, National Taiwan University, Taipei, Taiwan 10617 Republic of China \\ E-mail: fistung@hep1.phys.ntu.edu.tw
}

\begin{abstract}
We performed a search for a light pseudoscalar particle $X$ in the decay $K_{L}^{0} \rightarrow \pi^{0} \pi^{0} X, X \rightarrow \gamma \gamma$ with the E391a detector at KEK. Such a particle with a mass of $214.3 \mathrm{MeV} / c^{2}$ was suggested by the HyperCP experiment. We found no evidence for $X$ and set an upper limit on the product branching ratio for $K_{L}^{0} \rightarrow \pi^{0} \pi^{0} X, X \rightarrow \gamma \gamma$ of $2.4 \times 10^{-7}$ at the $90 \%$ confidence level. Upper limits on the branching ratios in the mass region of $X$ from 194.3 to $219.3 \mathrm{MeV} / c^{2}$ are also presented. The results had been published in [1].
\end{abstract}

2009 KAON International Conference KAON09,

June 09 - 122009

Tsukuba, Japan

* Speaker.

${ }^{\dagger}$ E391a Collaboration : Y. C. Tung, Y. B. Hsiung, M. L. Wu, K. F. Chen, J. K. Ahn, Y. Akune, V. Baranov, J. Comfort, M. Doroshenko, Y. Fujioka, T. Inagaki, S. Ishibashi, N. Ishihara, H. Ishii, E. Iwai, T. Iwata, I. Kato, S. Kobayashi, T. K. Komatsubara, A. S. Kurilin, E. Kuzmin, A. Lednev, H. S. Lee, S.Y. Lee, G.Y. Lim, J. Ma, T. Matsumura, A. Moisseenko, H. Morii, T. Morimoto, T. Nakano, H. Nanjo, J. Nix, T. Nomura, M. Nomachi, R. Ogata, H. Okuno, K. Omata, G. N. Perdue, Podolsky, K. Sakashita, T. Sasaki, N. Sasao, H. Sato, T. Sato, M. Sekimoto, T. Shinkawa, Y. Sugaya, A. Sugiyama, T. Sumida, S. Suzuki, Y. Tajima, S. Takita, Z. Tsamalaidze, T. Tsukamoto, Y. Wah, H. Watanabe, M. Yamaga, T. Yamanaka, H.Y. Yoshida, Y. Yoshimura, and Y. Zheng 


\section{Introduction}

We reported the first search of the decay $K_{L}^{0} \rightarrow \pi^{0} \pi^{0} X, X \rightarrow \gamma \gamma$ using the Run-II data sample taken by the E391a detector at KEK-PS. The particle $X$ with predicted $m_{X}=214.3 \mathrm{MeV} / c^{2}$ was suggested by HyperCP [2] to explain an observation of a very rare baryon decay $\Sigma^{+} \rightarrow p \mu^{+} \mu^{-}$. In their observation, the dimuon invariant mass of 3 observed events fell in a narrow mass range within the resolution of detector. HyperCP reported this may indicate the decay proceeded via an intermediate state, $\Sigma^{+} \rightarrow p X, X \rightarrow \mu^{+} \mu^{-}$, with a $X$ mass of $214.3 \pm 0.5 \mathrm{MeV} / c^{2}$.

Nearly a year later, one $[3,4]$ suggested a sgoldstino interpretation for the HyperCP observation and predicted the particle $X$ may also show up in several $K_{L}^{0}$ decays. $K_{L}^{0} \rightarrow \pi^{0} \pi^{0} X$ is one of the those predicted decays with an upper estimate of the branching ratio, $\operatorname{BR}\left(K_{L}^{0} \rightarrow \pi^{0} \pi^{0} X\right)=$ $1.2 \times 10^{-4}$. This gives us a chance to search for the light pseudoscalar particle, $X$, in the decay $K_{L}^{0} \rightarrow \pi^{0} \pi^{0} X, X \rightarrow \gamma \gamma$ with the E391a detector.

\section{Event Reconstruction}

Since the branching ratio of $X$ is highly saturated by the channel $X \rightarrow \gamma \gamma$, the decay $K_{L}^{0} \rightarrow$ $\pi^{0} \pi^{0} X$ is first signatured by six photons in the final state. In known $K_{L}^{0}$ decays, the decay $K_{L}^{0} \rightarrow 3 \pi^{0}$ with large $\operatorname{BR}\left(K_{L}^{0} \rightarrow 3 \pi^{0}\right)=19.56 \pm 0.14 \%$ has the same signature as the decay $K_{L}^{0} \rightarrow \pi^{0} \pi^{0} X$, separation of these decays was cruial in this study.

To reconstruct $K_{L}^{0} \rightarrow \pi^{0} \pi^{0} X$, we first selected events with six photonlike clusters on the CsI calorimeter without any in-time hits on other detectors. Afterwards, event reconstruction proceeded by solving for the decay vertex, assuming the $\pi^{0}$ mass and constraining the vertex to lie along the beam axis. The fiducial decay Z-vertex $\left(Z_{v t x}\right)$ region was defined to be between 250 and $550 \mathrm{~cm}$. There were 45 possible combinations to select two photon pairs from 6 photons to form two $\pi^{0}$ 's. The most likely pairing was chosen by a minimum $\chi_{z}^{2}$, which was calculated based on the difference between reconstructed $Z_{v t x}$ 's of the $\pi^{0}$ 's. In the $K_{L}^{0} \rightarrow \pi^{0} \pi^{0} X$ event, the $X$ mass was the invariant mass of the third photon pair $\left(M_{56}\right)$ and was reconstructed from the energy and hit positions of the remaining two photons and the $Z_{v t x}$ determined from two $\pi^{0}$ 's. The event reconstruction was further optimized by a constrained fit [5] requiring: (a) six photons to have the $K_{L}^{0}$ mass, (b) two photon pairs to have the $\pi^{0}$ mass, and (c) the $K_{L}^{0}$ momentum vector from the target to point to the center of energy of the photons on the calorimeter. The $\chi^{2}$ of the constrained fit $\left(\chi_{\text {fit }}^{2}\right)$ was calculated for all 45 combinations, and the one with the minimum $\chi_{\text {fit }}^{2}$ was chosen to be the correct pairing. For successful reconstructions, $M_{56}$ equals the $\pi^{0}$ mass for $K_{L}^{0} \rightarrow 3 \pi^{0}$ decays and the $X$ mass $\left(m_{X}\right)$ for $K_{L}^{0} \rightarrow \pi^{0} \pi^{0} X$ decays. Figure 1 shows $M_{56}$ of data, the $K_{L}^{0} \rightarrow 3 \pi^{0} \mathrm{MC}$ and the $K_{L}^{0} \rightarrow \pi^{0} \pi^{0} X \mathrm{MC}$ with a $214.3 \mathrm{MeV} / c^{2} X$ mass. The signal region is defined to be $211.3 \mathrm{MeV} / c^{2}<M_{56}<217.3$ $\mathrm{MeV} / c^{2}$. Since the $K_{L}^{0} \rightarrow 3 \pi^{0}$ decay has three $\pi^{0}$ 's in the final state, $M_{56}$ could be the invariant mass of any one of the three $\pi^{0}$ s. Since only the reconstructed masses of the first and second photon pairs are constrained, the pairing with the minimum $\chi_{\text {fit }}^{2}$ left the worst reconstructed mass of $\pi^{0}$ in the third photon pair. This explains the dip in the $\pi^{0}$ peak.

\section{Background Study}

So far as we know, the decays from $K_{L}^{0}$ with six photons in the final state are relatively clean 


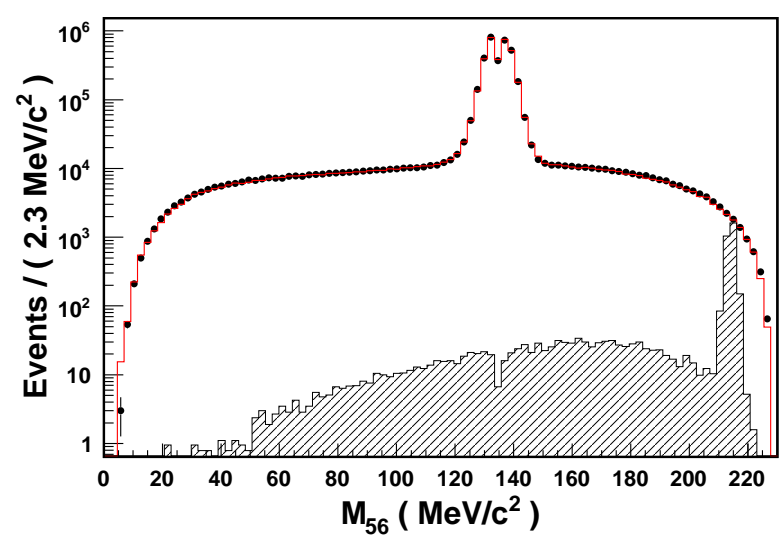

Figure 1: (color online) The $M_{56}$ distribution of the events with six photons in the calorimeter. The points with error bars show the data, and the red (open) histogram shows the $K_{L}^{0} \rightarrow 3 \pi^{0} \mathrm{MC}$ normalized to the number of data events. The shaded histogram represents the $K_{L}^{0} \rightarrow \pi^{0} \pi^{0} X \mathrm{MC}\left(m_{X}=214.3 \mathrm{MeV} / c^{2}\right)$ and was normalized assuming that $B R\left(K_{L}^{0} \rightarrow \pi^{0} \pi^{0} X, X \rightarrow \gamma \gamma\right)$ is $1.2 \times 10^{-4}$.

modes. In the large amount of the MC study, the background of the $K_{L}^{0} \rightarrow \pi^{0} \pi^{0} X$ decay was mainly from the $K_{L}^{0} \rightarrow 3 \pi^{0}$ decays. The MC study showed a long tail of $M_{56}$ of the $K_{L}^{0} \rightarrow 3 \pi^{0}$ events extended into the $X$ mass region. This tail was due to the bad measurement of the photon energy and position, and caused the wrong photon pairing combinations.

This background was suppressed by reconstructing over all photon combinations to the $K_{L}^{0} \rightarrow$ $3 \pi^{0}$ decay and discarding the event whose distribution was consistent with the $K_{L}^{0} \rightarrow 3 \pi^{0}$ decay. We applied another constrained fit, called the "full constrained fit", by requiring the three photon pairs to have $m_{\pi^{0}}$, and reconstructing each event as $K_{L}^{0} \rightarrow 3 \pi^{0}$. As shown in Fig. 2, the smaller fitted $\chi_{\text {full }}^{2}$ indicated the higher probability the event from the $K_{L}^{0} \rightarrow 3 \pi^{0}$ decay. By discarding events with $\chi_{\text {full }}^{2}<50$, the wrong pairing background events in the region $M_{56}>165 \mathrm{MeV} / \mathrm{c}^{2}$ were suppressed by a factor $6.6 \times 10^{2}$. The acceptance of $K_{L}^{0} \rightarrow \pi^{0} \pi^{0} X\left(m_{X}=214.3 \mathrm{MeV} / c^{2}\right)$ decays by the $\chi_{\text {full }}^{2}$ cut was estimated to be $61.0 \%$ based on the simulations.

\section{Signal Extraction}

After all selection cuts applied on data, only 2 events survived in the signal region (211.3217.3 MeV/c $c^{2}$ ) of $m_{X}=214.3 \mathrm{MeV} / c^{2}$. To estimate the number of background events in the signal region, we used the data events in the sideband regions $\left(165 \mathrm{MeV} / c^{2}<M_{56}<211.3 \mathrm{MeV} / c^{2}\right.$ and $\left.M_{56}>217.3 \mathrm{MeV} / \mathrm{c}^{2}\right)$. Since the signal region is near the edge of the $M_{56}$ tail, the background shape was modeled with the ARGUS function [6], which is defined as:

$$
f(x)_{A R G U S}=x \sqrt{1-\left(\frac{x}{M_{e p}}\right)^{2}} \exp \left[\alpha-\alpha\left(\frac{x}{M_{e p}}\right)^{2}\right],
$$

where $M_{e p}$ is the end point of the ARGUS function, $x$ is $M_{56}$, and $\alpha$ determines the curvature of ARGUS function. For the signal MC, the reconstructed mass peak is asymmetric because of 


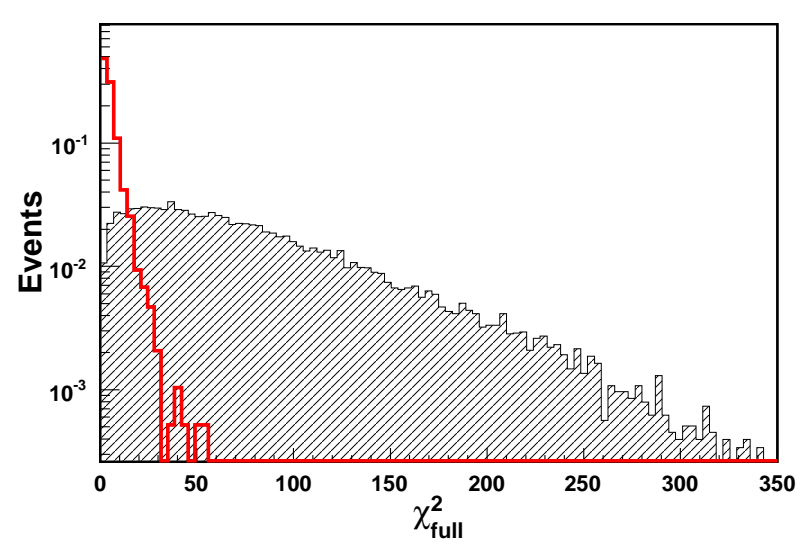

Figure 2: (color online) The $\chi_{\text {full }}^{2}$ distribution of events just before the $\chi_{\text {full }}^{2}$ cut, explained in the text, in the signal region of $m_{X}=214.3 \mathrm{MeV} / c^{2}$. The red (open) histogram shows the $K_{L}^{0} \rightarrow 3 \pi^{0} \mathrm{MC}$ and shaded histogram shows the $K_{L}^{0} \rightarrow \pi^{0} \pi^{0} X \mathrm{MC}\left(m_{X}=214.3 \mathrm{MeV} / c^{2}\right)$. The cut point was set at 50 .

the kinematical limit of the $X$ mass. In this case, a double Gaussian function with different mean values of the two composition Gaussian functions was chosen as the probability density function (PDF) to model the signal shape from the signal MC. Afterwards, the number of signal events was extracted by the unbinned extended-likelihood method. Fig. 3 shows the modeling result of the 214.3 MeV/c $c^{2} X$ mass. The dip in the signal region implied there is no evidence of $X$.

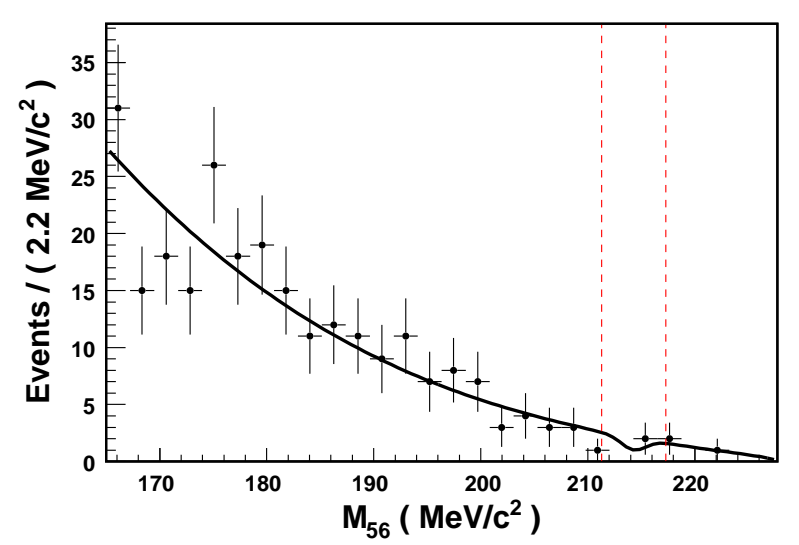

Figure 3: The modeling result of the $214.3 \mathrm{MeV} / \mathrm{c}^{2} X$ mass. Error bars show the data, and the solid line which is the combination of background and signal PDF, is the modeling result. The region between two dashed lines is the signal mass region.

\section{Results}

In this study, we presented the search of $X$ in the mass region from 194.3 to $219.3 \mathrm{MeV} / \mathrm{c}^{2}$. For the search of $X$ in the mass region, the results were not stable, thus were not shown. The branching 
ratio $(\mathrm{BR})$ was calculated by:

$$
B R\left(K_{L}^{0} \rightarrow \pi^{0} \pi^{0} X\right)=\frac{N_{s}}{\text { Acceptance } \cdot N\left(K_{L}^{0} \text { decays }\right)},
$$

where the acceptance was the signal acceptance calculated using the signal MC events. The $K_{L}^{0}$ flux, $N\left(K_{L}^{0}\right.$ decays $)$, was estimated by the $K_{L}^{0} \rightarrow 3 \pi^{0}$ mode and was cross-checked by the $K_{L}^{0} \rightarrow \pi^{0} \pi^{0}$ mode. The $K_{L}^{0}$ flux at $10 \mathrm{~m}$ from the target was determined to be $(1.32 \pm 0.04) \times 10^{11}$ based on the number of decays downstream of that point. In the purpose of systematical error cancellation, the selection cuts, applied for the $K_{L}^{0}$ flux estimation, are mostly the same as for the analysis, except for the $\chi_{\text {full }}^{2}$ cut, which rejected the $K_{L}^{0} \rightarrow 3 \pi^{0}$ events.

The study showed no evidence of $X$ was found. The upper limit of branching ratio was calculated by integrating up to $90 \%$ of the area under the likelihood function, for $N_{s}>0$. The results of different $m_{X}$ are summarized in Table 1.

Table 1: A summary of the signal yields $\left(N_{s}\right)$, the acceptances of the signal decay (Accept.), central value of the branching ratios (B.R.), and the upper limits at the $90 \%$ confidence level(U.L.).

\begin{tabular}{|c|c|c|c|c|}
\hline$\overline{m_{X}\left(\mathrm{MeV} / c^{2}\right)}$ & $N_{s}$ & Accept. $\left(10^{-4}\right)$ & B.R. $\left(10^{-8}\right)$ & " U.L. $\left(10^{-7}\right)$ \\
\hline 194.3 & $6.4_{-5.2}^{+6.0}$ & $1.09 \pm 0.01$ & $44.5_{-36.4}^{+41.6}($ stat. $) \pm 8.4($ syst. $)$ & 10.7 \\
\hline 199.3 & $3.5_{-4.0}^{+4.8}$ & $1.09 \pm 0.01$ & $24.1_{-27.8}^{+33.2}($ stat. $) \pm 10.0($ syst.$)$ & 7.9 \\
\hline 204.3 & $-0.7_{-2.7}^{+3.4}$ & $1.09 \pm 0.01$ & $-4.9_{-18.8}^{+23.9}($ stat. $) \pm 16.3$ (syst. $)$ & 5.0 \\
\hline 209.3 & $-1.5_{-1.8}^{+2.5}$ & $1.08 \pm 0.01$ & $-10.7_{-12.3}^{+17.2}($ stat. $) \pm 11.6($ syst. $)$ & 3.5 \\
\hline 214.3 & $-1.4_{-0.9}^{+1.7}$ & $1.08 \pm 0.01$ & $-10.0_{-6.6}^{+11.8}($ stat. $) \pm 5.6($ syst. $)$ & 2.4 \\
\hline 219.3 & $-0.3_{-1.0}^{+1.7}$ & $1.09 \pm 0.01$ & $-2.4_{-6.5}^{+0.0}($ stat. $) \pm 1.9$ (syst. $)$ & 2.6 \\
\hline
\end{tabular}

The upper limit on the branching ratio for $K_{L}^{0} \rightarrow \pi^{0} \pi^{0} X, X \rightarrow \gamma \gamma$ decay depends on the lifetime $\left(\tau_{X}\right)$ and the mass of $X$ as shown in Fig. 4. The upper limits were independent of the $X$ lifetime if it was shorter than $10^{-10} \mathrm{~s}$. The upper limit on the branching ratio for $K_{L}^{0} \rightarrow \pi^{0} \pi^{0} X$, $X \rightarrow \gamma \gamma\left(m_{X}=214.3 \mathrm{MeV} / c^{2}\right)$ decay was set to be $2.4 \times 10^{-7}$ in the lifetime region of $\tau_{X}<10^{-10} \mathrm{~s}$. The upper limits weaken by a factor of 2-3 if the $X$ lifetime was $10^{-9} \mathrm{~s}$.

\section{References}

[1] E391a collaboration, Y. C. Tung et al., Phys. Rev. Lett. 102, 051802 (2009).

[2] HyperCP collaboration, H. K. Park et al., Phys. Rev. Lett. 94, 021801 (2005).

[3] D.S. Gorbunov and V.A. Rubakov, Phys. Rev. D73, 035002 (2006).

[4] D.S. Gorbunov and V.A. Rubakov, Phys. Rev. D64, 054008 (2001).

[5] P. Avery, Fitting Theory I-V. http://www.phys.ufl.edu/ avery/fitting.html.

[6] ARGUS collaboration, H. Albrecht, Phys. Lett B 241, 278 (1990). 


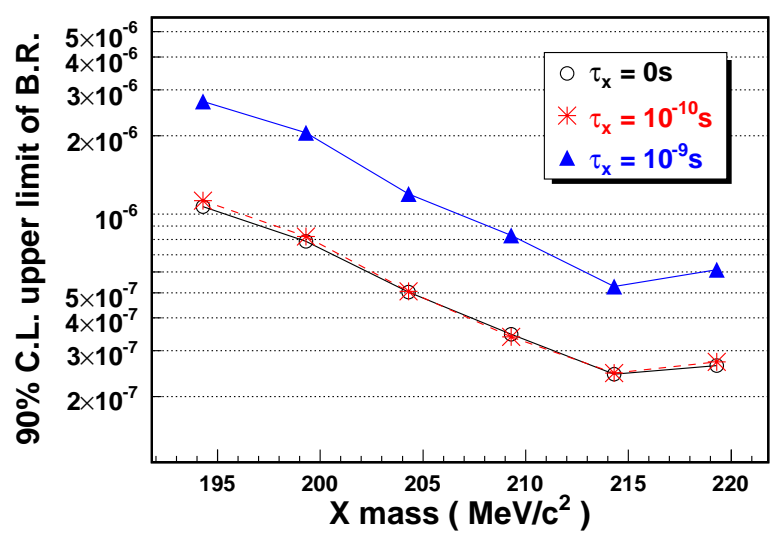

Figure 4: (color online) The $90 \%$ confidence level upper limits of the $\mathrm{BR}\left(K_{L}^{0} \rightarrow \pi^{0} \pi^{0} X, X \rightarrow \gamma \gamma\right)$ for different $X$ lifetimes as a function of the $X$ mass. 